\title{
Rayleigh scattering in fused silica samples for gravitational wave detectors
}

\author{
Xu Chen ${ }^{a}, *$, Li Ju ${ }^{a}$, Raffaele Flaminio ${ }^{b}$, Harald Lück ${ }^{c}$, Chunnong Zhao ${ }^{\text {a }}$, David G. Blair ${ }^{\text {a }}$ \\ a School of Physics, University of Western Australia, 35 Stirling Hwy, Crawley, WA6009, Australia \\ ${ }^{\mathrm{b}}$ Laboratoire des Matériaux Avancés, IN2P3/CNRS, Campus de la DOUA, 7, Avenue Pierre de Coubertin, 69622, Villeurbanne Cedex, France

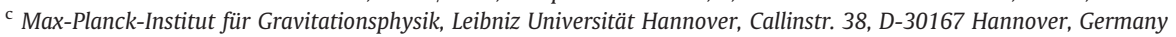

\section{A R T I C L E I N F O}

\section{Article history:}

Received 18 January 2011

Received in revised form 7 June 2011

Accepted 8 June 2011

Available online 23 June 2011

\section{Keywords:}

Rayleigh scattering

Fused silica

Polarisation

Homogeneity

Gravitational wave detectors

\begin{abstract}
A B S T R A C T
Laser interferometer gravitational wave detectors require very high optical quality test masses. We report the bulk Rayleigh scattering in high quality fused silica samples. Results show that the scattering of the high quality fused silica is similar for various grades of fused silica from Heraeus. The total integrated scattering is about $0.7 \mathrm{ppm} \mathrm{cm}^{-1}$ at $1064 \mathrm{~nm}$ wavelength, which agrees with the theoretical value calculated using known fused silica parameters. All samples show Rayleigh scattering ratio inhomogeneity of $\sim 4 \%$.
\end{abstract}

(c) 2011 Elsevier B.V. All rights reserved.

\section{Introduction}

Laser interferometer gravitational wave detectors need an extremely high sensitivity in order to detect a fractional length change of the order of $10^{-22}$ in kilometre scale optical cavities. The planned advanced detectors will use very high optical powers to reduce shot noise. In laser interferometric gravitational wave detectors, the laser passes through several components of the interferometer such as the beam-splitter, the power recycling mirror and the input mirrors. These detectors will require test masses with very high optical quality-very low absorption to reduce thermal lensing [1] and very low optical scattering. The current and planned advanced room temperature gravitational wave detectors use fused silica as their test mass material. Great care has been taken to ensure very low optical absorption $<1 \mathrm{ppm} \mathrm{cm}^{-1}[2]$ in the critical optical components. There is a risk that optical scattering could cause loss of sensitivity through scattered light being Doppler shifted from vibrating components and re-entering the measurement beam. Whereas surface scattering can be taken care of by providing a good polish and a good coating, bulk scattering cannot be lowered below the intrinsic Rayleigh scattering level even in very homogeneous substrates. Previous measurements reported scattering at the high end of acceptable values [3]. For this reason we undertook of measurements of Rayleigh scattering in bulk high quality fused silica samples similar to those used in gravitational wave detectors. The measurements were performed by the Automatic Rayleigh Scattering Mapping Systems (ARSMS) [4] developed at University of Western Australia. The device uses a cooled CCD camera to image scattered light from beams passing

\footnotetext{
* Corresponding author. Tel.: +61 864884552.

E-mail address: chenxu@cyllene.uwa.edu.au (X. Chen).
}

through bulk samples. Careful new calibration shows that previous results in reference [3] are erroneous. With new calibration the materials tested have scattering values close to the theoretical scattering expected for fused silica. In this paper we first summarise the standard theory of Rayleigh scattering, to explore how the field scattering coefficient is defined. Then we describe the experimental results in terms of single beam, polarisation angle dependence and observation angle dependence, which confirm that the scattering follows the Rayleigh law. The homogeneity of the samples is also investigated.

\section{Rayleigh scattering}

In a solid material Rayleigh scattering occurs when there is some inhomogeneity or small defects in the material. In high quality materials, the scattering is mainly due to the fluctuation of the density of the material. Theoretical value of the scattering is described as the scattering coefficient $\alpha_{\text {sca }}$ (the attenuation coefficient per unit length) in $\mathrm{ppm} \mathrm{cm}^{-1}[5]$

$\alpha_{s c a}=\frac{8}{3} \frac{\pi^{3}}{\lambda^{4}}\left(n^{4} p\right)^{2} k T \beta_{T}$

where $\lambda$ is wavelength, $n$ is the index of refraction, $p$ is the photoelastic constant, $k$ is the Boltzmann constant, $T$ is absolute temperature and $\beta_{T}$ is the isothermal compressibility of the material. The expression given in Eq. (1) is based on the assumption of thermodynamic equilibrium, which is valid for liquids. The random structure of fused silica is not determined by the ambient temperature, but by the fused silica transition temperature at which the fused silica becomes solid [6]. Using known fused silica parameters: 


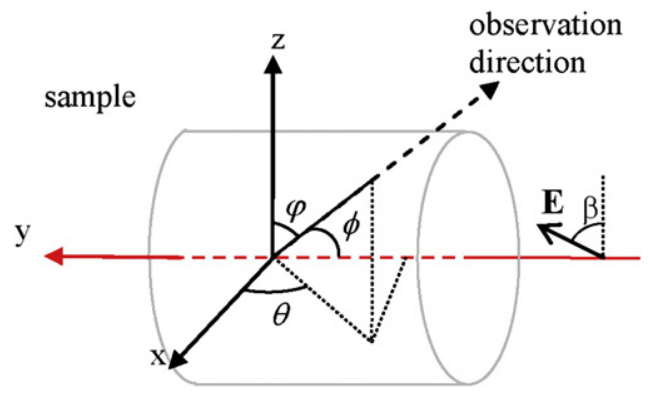

Fig. 1. Diagram of the definitions of the polarisation direction of incident light and angles for observation direction: $\beta$ is the angle between polarisation direction and the vertical axis ( $z$-axis), $\theta$ is the angle between observation direction and incident light in horizontal plane ( $x-y$ plane), $\varphi$ is the angle between observation direction and $z$-axis and $\phi$ is the angle between observation direction and the incident light.

$n=1.4498, \quad p=\left(p_{11}+2 p_{12}\right) / 3, \quad p_{11}=0.121$ and $p_{12}=0.270$, $\mathrm{T}=1700 \mathrm{~K}, \beta_{\mathrm{T}}=7 \times 10^{-11} \mathrm{~m} \mathrm{~s}^{2} \mathrm{~kg}^{-1}$ and $\lambda=1064 \mathrm{~nm}$, the theoretical value $\alpha_{\mathrm{sc}}=1.0 \mathrm{ppm} \mathrm{cm}^{-1}$.

Experimentally, the Rayleigh scattering can be obtained by measuring the scattered light at different angles. The intensity of scattering differs with different polarisation directions of the incident light and observation directions. In general, the scattering ratio $R_{p}$ for vertically polarised light and an arbitrary observation direction is given by [7]

$$
\begin{aligned}
R_{p}=\frac{I_{s}}{I_{\text {in }}}= & R_{p, 90}\left(\cos ^{2} \varphi \sin ^{2} \beta-\frac{1}{2} \sin (2 \beta) \cos \theta \sin 2 \varphi\right. \\
& \left.+\left(\cos ^{2} \beta+\sin ^{2} \beta \sin ^{2} \theta\right) \sin ^{2} \varphi\right) .
\end{aligned}
$$

where $I_{s}$ is intensity of scattered light per solid angle, $I_{\text {in }}$ is intensity of incident light, $\beta$ is the angle between the polarisation direction and the vertical axis ( $z$-axis), $\theta$ is the angle between observation direction and incident light in horizontal plane ( $x-y$ plane) and $\varphi$ is the angle between observation direction and z-axis, as shown in Fig. 1. The term $R_{p, 90}$ is the maximum scattering ratio which is obtained when light polarisation direction is perpendicular to the observation angle, such that, $\beta=0, \theta=0$ and $\varphi=\pi / 2$.

The scattering coefficient $\alpha_{\text {sca }}$ is equal to the integrated total scattering ratio. The integrated total scattering ratio of an isotropic material with a polarised incident light can be obtained by integrating Eq. (2) over the total scattering surface. The scattering coefficient $\alpha_{\text {sca }}$ is then:

$\alpha_{s c a}=\frac{I_{s t}}{I_{\text {in }}}=\int_{0}^{\pi} \int_{0}^{2 \pi} R_{p} \sin \varphi d \theta d \varphi=\frac{8}{3} \pi R_{p, 90}$.

Here $I_{s t}$ is the total scattering intensity.

\section{Experiment setup}

Light scattering measurements were performed by an Automatic Rayleigh Scattering Mapping Systems (ARSMS) setup shown in Fig. 2.

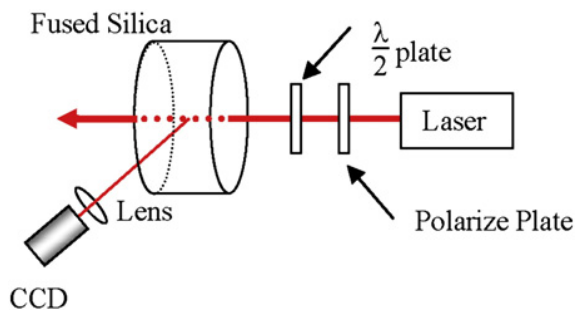

Fig. 2. Experimental set up for scattering measurements. A He-Ne laser passes through a polariser and a half-wave plate which is used to vary the polarisation direction of the incident laser beam. The laser beam enters a flat polished surface of the sample. The scattered light is recorded by a CCD camera. The sample is mounted on a precision XYZ $\phi$ platform for scanning the whole volume of the sample.

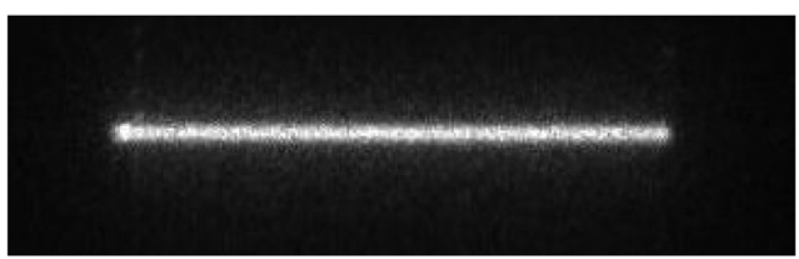

Fig. 3. A laser beam scattering image taken by a high-sensitivity CCD camera.

This device has been used to measure the scattering in sapphire test masses which revealed ghost images of the crystallisation due to the crystal growth process [7]. The instrument is sensitive down to levels $<10^{-3} \mathrm{ppm} \mathrm{cm}^{-1}$. We used a $\mathrm{He}-\mathrm{Ne}$ laser $(\lambda=633 \mathrm{~nm})$ because our CCD camera has poor sensitivity at $1064 \mathrm{~nm}$, which is the laser wavelength of current gravitational wave detectors. However, it is still viable to use an Nd:YAG laser to verify that the scattering follows the Rayleigh scattering rule [3]:

$R \propto \lambda^{-4}$

The results reported here have been scaled to the value expected at $1064 \mathrm{~nm}$ wavelength.

The scattered light was recorded by a cooled astronomical CCD camera (SBIG ST-402MECCD Imager). The flat surfaces of the fused silica samples are perpendicular to the incident light. The polarisation direction of the incident beam was changed by a half-wave plate. The sample is mounted on a precision XYZ $\phi$ platform so that the scattering of the whole volume of the sample can be scanned. The samples had polished walls to allow imaging of the probe beams and results are corrected for the magnification of the beam image by the cylindrical lens of the wall, and care is taken to eliminate the effects of surface scattering. Full details are given in reference [8].

The data were recorded in digital images as shown in Fig. 3. They were then processed to obtain an average scattering intensity, by averaging the intensity of a single-beam scattering in the image, as described in reference [8]. For the homogeneous study, we scanned the whole sample and assemble an array of single beam data to obtain the $2 \mathrm{D}$ or $3 \mathrm{D}$ images.

In order to reduce the background noise the apparatus was located in a dark room and black cloth was used to prevent light scattering from all reflective surfaces. Most recordings were taken automatically. The exposure time was $2 \mathrm{~s}$ for every image.

\section{Experimental results}

Four fused silica samples were measured. Samples 1 and 2 are Heraeus Suprasil@ 3001 and 3002 cubic samples $\left(30 \times 30 \times 30 \mathrm{~mm}^{3}\right)$ provided by the GEO project[9]. Sample 3 is a Heraeus Suprasil 312 cylindrical sample $\left(\phi 50 \times 20 \mathrm{~mm}^{3}\right)$ provided by Laboratoire des Matériaux Avancés, the VIRGO project [10]. Sample 4 is an old

Table 1

Calibration of Rayleigh scattering ratio of four samples at $1064 \mathrm{~nm}$.

\begin{tabular}{llll}
\hline Material & $\begin{array}{l}\text { Measured } \\
\text { (counts) }\end{array}$ & $\begin{array}{l}\text { Pike et al. [11] } \\
\left(10^{-2} \mathrm{ppm} \mathrm{cm}^{-1} \Omega^{-1}\right)\end{array}$ & $\begin{array}{l}\text { Calibrated scattering } \\
\left(10^{-2} \mathrm{ppm} \mathrm{cm}^{-1} \Omega^{-1}\right)\end{array}$ \\
\hline Background & 3.8 & & $0.07 \pm 0.01$ \\
Empty tube & 32 & & $0.55 \pm 0.06$ \\
Distilled water & 739 & 11.52 & $12.8 \pm 1.4$ \\
Toluene & 9006 & 170.2 & $156 \pm 17$ \\
Methanol & 1962 & 34.95 & $33.9 \pm 3.8$ \\
Sample 4 & 460 & & $8.0 \pm 0.9$ \\
\hline
\end{tabular}

Note: Pike et al's results have been converted for the differences in wavelength using Eq. (4). The results in column 4 are the averages obtained by using the values in column 3 as calibrator separately. 

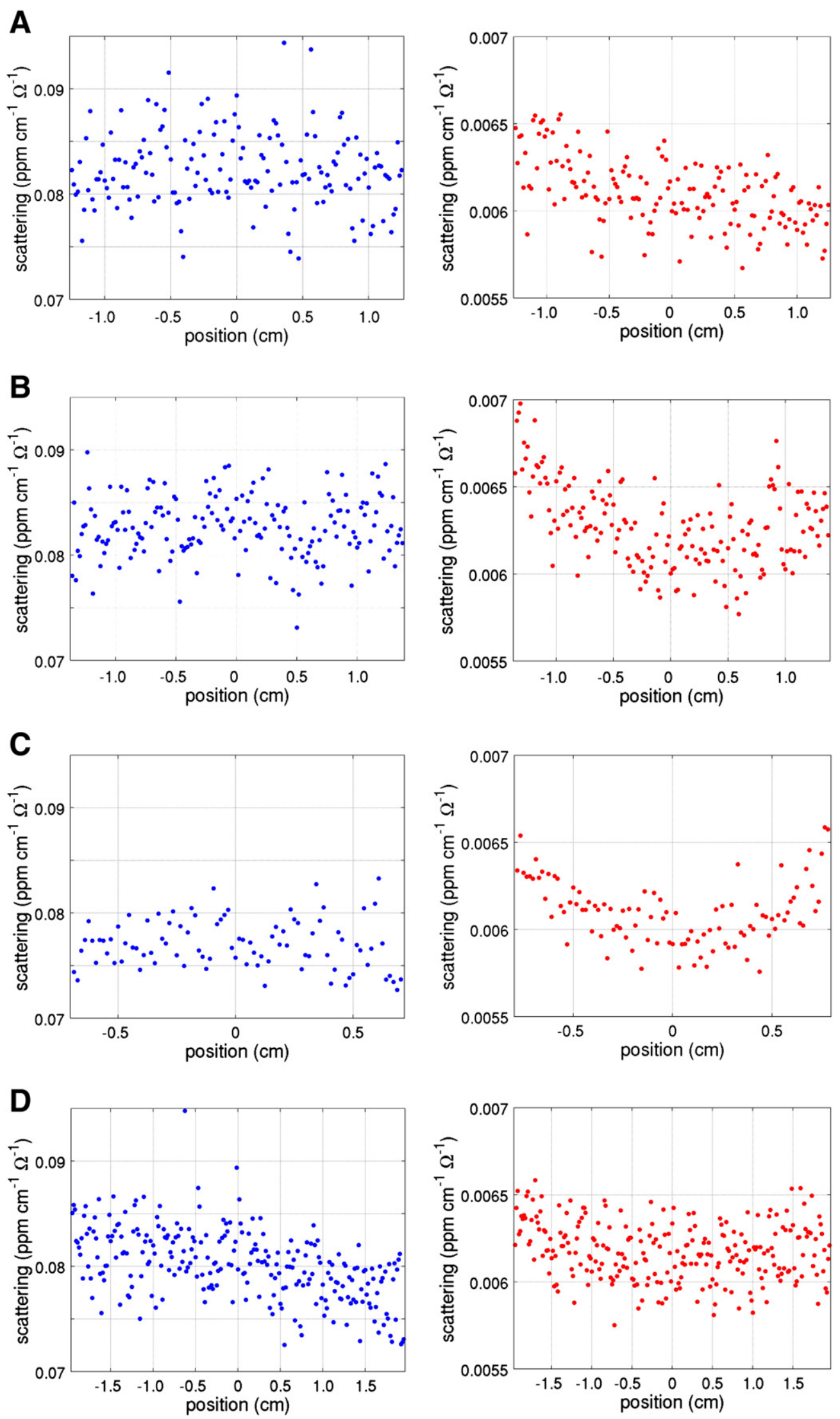

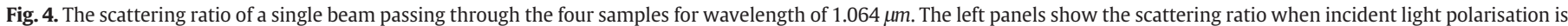

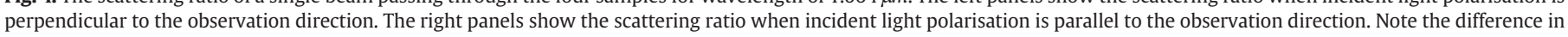
length of these samples. 
Heraeus Suprasil SV (Special Virgo) cylindrical sample of $\phi 50 \times 100 \mathrm{~mm}^{3}$ provided by the VIRGO project 10 years ago.

All four samples show similar scattering levels. The scattered light is quite uniform along the length of samples. The intensity of the scattering changes with the incident light polarisation direction relative to the observation angle as expected. The average maximum scattering ratio is $0.08 \mathrm{ppm} \mathrm{cm}^{-1} \Omega^{-1}$ at $1064 \mathrm{~nm}$ ( $\Omega$ is the observation solid angle), resulting in a scattering coefficient of $\alpha_{\text {sca }} \sim 0.7 \mathrm{ppm} \mathrm{cm}^{-1}$. The homogeneity of the samples is also investigated by scanning the cross sections of the samples.

\subsection{Calibration}

We used materials with previously known scattering ratio to calibrate our results. Multiple measurements with various samples were used to ensure that errors due to contamination are minimised. Three nominal pure materials were used: distilled water, toluene and methanol. Table 1 summarises the calibration results. Column 2 of this table shows the scattering amplitude in the unit of the count of the CCD camera. Column 3 is the results of Pike et al. [11]. Using Pike's results we can obtain a calibration coefficient in the unit of ppm cm ${ }^{-1} \Omega^{-1}$ per count for each material. The average of these 3 coefficients, $(1.73 \pm 0.16) \times 10^{-2} \mathrm{ppm} \mathrm{cm}{ }^{-1} \Omega^{-1}$ per count, is then used to determine the scattering ratio of our samples shown in Column 4. The calibrated scattering ratio of one silica sample
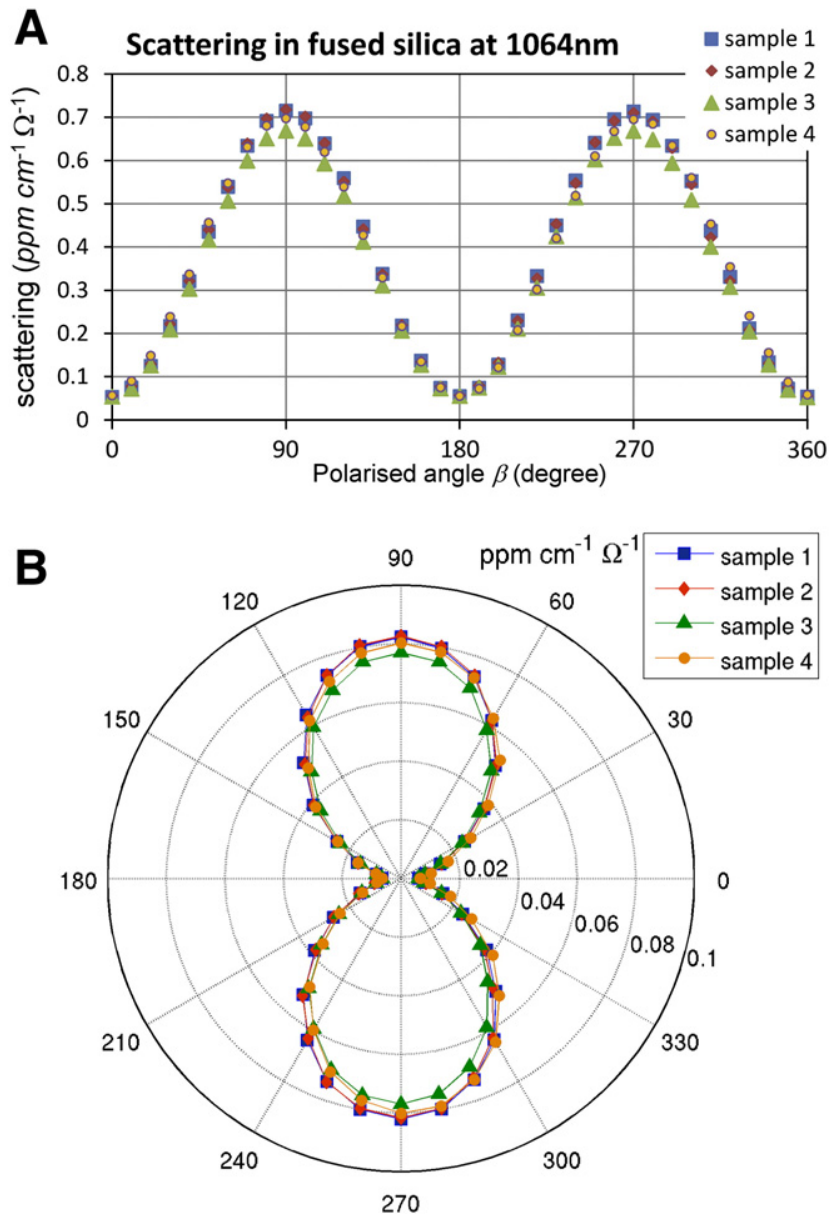

Fig. 5. (A) Right angle scattering ratios of the 4 samples vs. incident light polarisation direction. The angle $\beta$ is the angle between the polarisation direction and the observation plan (the plan of the observation direction and the incident light). (B) Scattering ratios in polar coordinate.

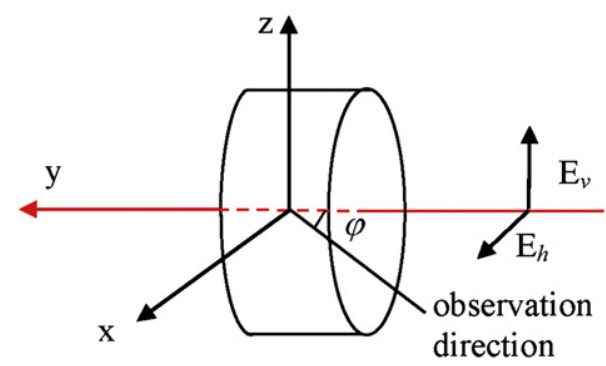

Fig. 6. Scattering measurement set-up with changing observation angle. $\varphi$ is the angle between observation direction and the incident beam.

(sample 4) is also shown. The systematic error from the calibration coefficient is about $10 \%$ whilst measurement error between identical samples was less than $5 \%$.

Also listed in Table 1 are the scattering ratio of light through air and the scattering ratio of light through an empty $\phi 30 \times 100 \mathrm{~mm}^{3}$ glass tube which was used to hold the liquid samples. The purpose of these measurements was to estimate the light scattered by air and surrounding environment. It can be seen that the noise level is negligible. All scattering measurements in this paper include noise due to background light. The count rate due to dark current was measured by completely covering the CCD camera and has been subtracted from the raw measurements.

All data presented in the table refers to vertically polarised incident light. The scattering ratio of unpolarised light can be calculated using the formula

$R_{90, u}=\frac{1}{2} R_{90, v}\left(1+\rho_{u}\right)$,

where subscript $u$ and $v$ indicate unpolarised and vertically polarised incident light, respectively, and observed at $90^{\circ}$ relative to the incident beam. The parameter $\rho_{\mathrm{u}}$ is the depolarisation ratio of scattered light from an unpolarised incident beam [11-13].

\subsection{Single beam scattering}

Fig. 4 shows the right angle single beam scattering ratio along the length of the samples. The left panels show the scattering ratio when the incident light polarisation is perpendicular to the observation direction, whereas the right panels show scattering ratio when the incident light polarisation is parallel to the observation direction. It can be seen that the scattering for the 4 samples is all quite uniform

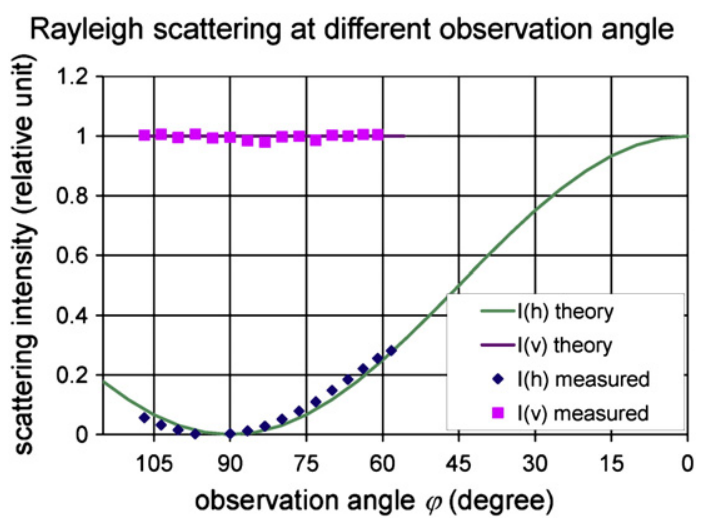

Fig. 7. Relative scattering intensity at different observation angles. The dots show measured data, whereas the solid lines are theoretical values given in Eq. (6). 
Table 2

Rayleigh scattering of four samples at $1064 \mathrm{~nm}$.

\begin{tabular}{llccc}
\hline Sample & 1 & 2 & 3 & 4 \\
\hline $\begin{array}{l}\text { Horizontal polarisation } \\
10^{-2} \mathrm{ppm} \mathrm{cm}^{-1} \Omega^{-1}\end{array}$ & 0.61 & 0.63 & 0.60 & 0.64 \\
$\begin{array}{c}\text { Vertical polarisation } \\
\left(\mathrm{R}_{\mathrm{p}, 90}\right) 10^{-2} \mathrm{ppm} \mathrm{cm} \mathrm{cm}^{-1} \Omega^{-1}\end{array}$ & 8.2 & 8.3 & 7.7 & 8.0 \\
$\begin{array}{c}\text { Total integrated scattering } \\
\left(\alpha_{\text {sca) }} 10^{-2} \mathrm{ppm} \mathrm{cm}^{-1}\right.\end{array}$ & 71.7 & 71.4 & 67.9 & 70.5 \\
\hline
\end{tabular}

samples 2 and 3 when the scattering level is low with incident light polarisation parallel to the observation direction. For both polarisations, the fractional scattering ratios are similar.
To confirm that the scattering measured here is truly Rayleigh scattering ratio, we measured the scattering as a function of the light polarisation and as a function of the observation angle.

\subsection{Right angle scattering vs polarisation direction of light}

For the right angle scattering, the camera was placed along $x$-axis $(\theta=0, \varphi=\Pi / 2)$. The scattering ratio can be simply expressed as

$$
R(\beta)=R_{p, 90} \cos ^{2}(\beta) .
$$

Since fused silica is an isotropic material, rotating the incident polarisation direction whilst observing at one fixed direction is equivalent to rotating the observation angle whilst the polarisation
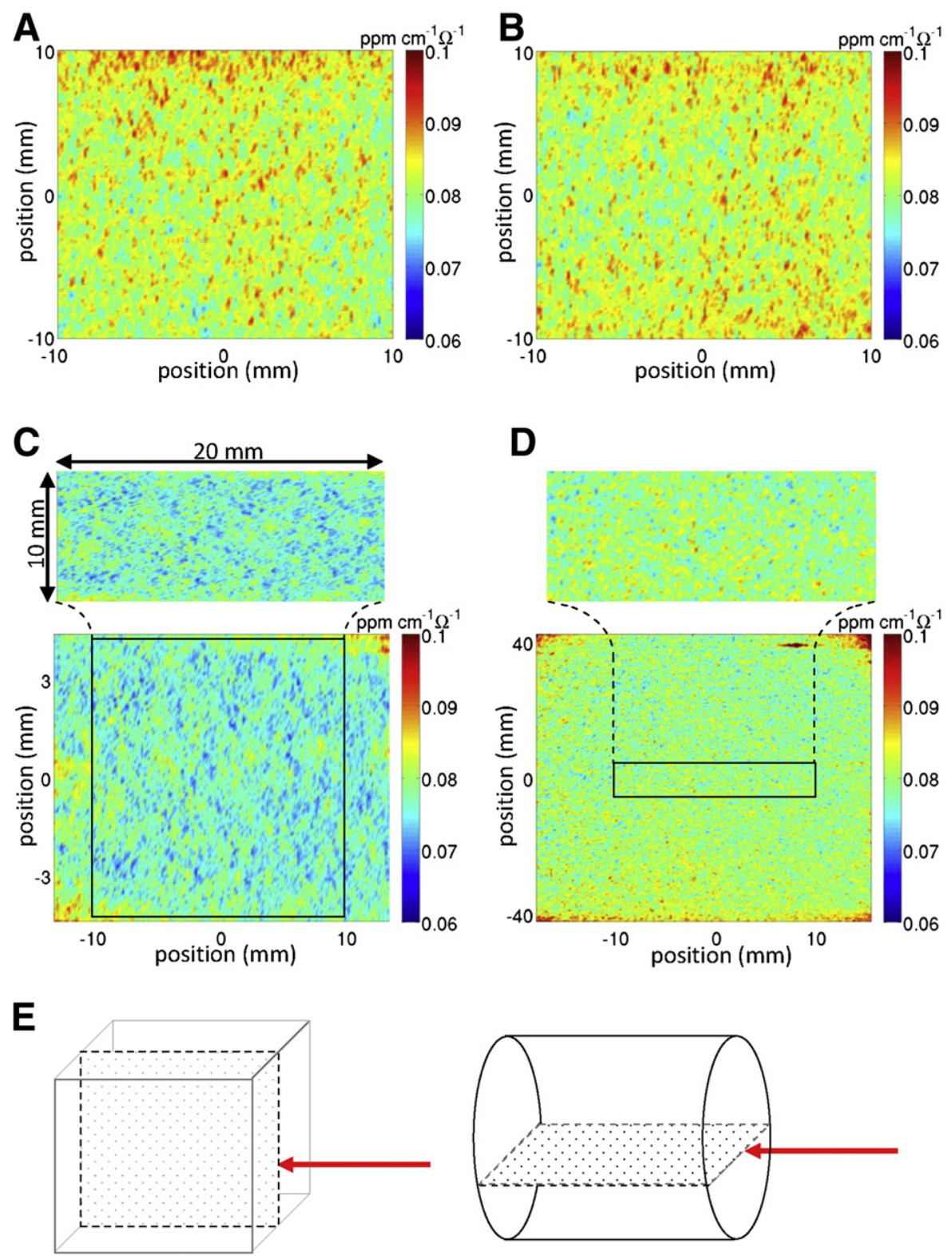

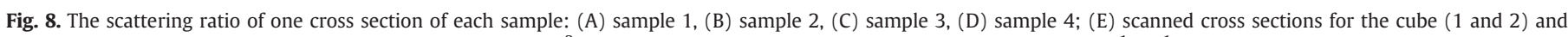

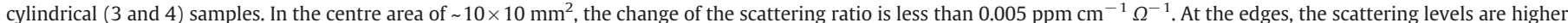

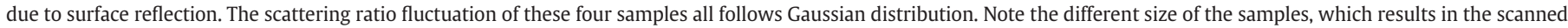

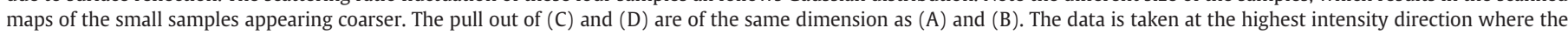
electrical field is perpendicular to the observation angle. 
Table 3

Homogeneity of the samples.

\begin{tabular}{lllll}
\hline Sample & 1 & 2 & 3 & 4 \\
\hline $\begin{array}{l}\text { Average scattering ratio } \\
\text { (cross section) }\end{array}$ & 0.082 & 0.083 & 0.077 & 0.080 \\
Variation & $4.1 \%$ & $4.4 \%$ & $4.6 \%$ & $4.4 \%$ \\
\hline
\end{tabular}

direction is fixed. So the angle $\beta$ is simply the angle between the observation direction and the incident polarisation direction. Fig. 5 (A) and (B) show the average single beam scattering ratio of the four samples as a function of $\beta$ in polar and linear coordinate respectively by rotating polarised direction of incident light. They all follow the $A \cos ^{2}(\beta)+C$ pattern as expected, where $A$ is equal to $R_{p, 90}$ and $C$ corresponds to scattering when incident light polarisation is parallel to the observation direction $(\beta=0)$. The non-zero scattering level of $C$ is believed to be due to the depolarised scattering [12]. The depolarisation ratio of the scattered light is defined as:

$\rho_{u}=R_{90, h} / R_{90, v}=2_{\rho_{v}} /\left(1+\rho_{v}\right)$,

where $\rho_{v}=R_{H 90, v} / R_{V 90, v}$.

The subscripts $h$ and $v$ denote horizontal and vertical polarisation of the incident light whilst $H$ and $V$ denote the horizontal and vertical polarised scattered light [13]. In our measurement, $\rho_{\mathrm{u}} \sim 0.08$, which agrees with Reference [12].

\subsection{Scattering vs observation direction}

It is well known that Rayleigh scattering also changes with the observation angle relative to the incident light. The observation direction was changed in $\mathrm{x}-\mathrm{y}$ plane and $\varphi$ is the angle between observation direction and incident light as shown in Fig. 6. The scattering ratio is then

$\frac{I_{h}}{I_{i}}=R_{p, 90} \cos ^{2} \varphi$
$\frac{I_{v}}{I_{i}}=R_{p, 90}$,

Here $h$ denotes that the polarisation direction is in $x-y$ plane and $v$ denotes the polarisation direction perpendicular to $x-y$ plane.

Although we could not measure the scattering with very small $\varphi$ due to the surface scattering of those small samples, we did confirm the scattering ratio is following the Rayleigh scattering law as shown in Fig. 7. Thus what we measured here is dominated by Rayleigh scattering.

\subsection{Total scattering}

Using the previous calibration and Eq. (3), we can obtain the scattering ratios and the scattering coefficients of the samples. Table 2 summarises measured scattering ratios in four samples with horizontally and vertically polarised incident light, as well as the calculated total scattering ratio (scattering coefficient $\alpha_{\text {sca }}$ ). It can be seen that the levels of the scattering are very similar for all 4 samples.

\subsection{Cross section homogeneity}

We also scanned the samples to evaluate the homogeneity of the samples. Fig. 8 shows cross sections of the scattering ratios of the 4 samples. The average scattering ratio is between 0.07 and $0.09 \mathrm{ppm} \mathrm{cm}^{-1} \Omega^{-1}$ indicated in Table 3 with sample 3 having the lowest average scattering. The variation near the centre area of the samples is less than $0.005 \mathrm{ppm} \mathrm{cm}^{-1} \Omega^{-1}$. Further analysis showed that scattering fluctuation of these four samples follows a Gaussian distribution with standard deviation ranging from $4.1 \%$ to $4.6 \%$. Near the edges, the scattering ratio is high due to surface reflections. The graininess of the scattering ratio appears to be inherent to the crystal and the patterns are repeatable. Results show that the inhomogeneity of the samples is $\sim 4 \%$.

\section{Conclusion}

We have measured the scattering ratio of four high quality fused silica samples. We have confirmed that the scattering ratio follows the angular dependence and polarisation dependence expected by the Rayleigh scattering law. All samples show similar scattering levels and inhomogeneity levels. The total integrated scattering ratio (scattering coefficient $\alpha_{\text {sca }}$ ) at $1064 \mathrm{~nm}$ is $\sim 0.7 \mathrm{ppm} \mathrm{cm}^{-1}$. This is comparable to the theoretical value of $1.0 \mathrm{ppm} \mathrm{cm}^{-1}$. This number expressed as $0.32 \mathrm{~dB} / \mathrm{km}$ is also comparable to the known attenuation in ultra pure FS fibre of $0.34 \mathrm{~dB} / \mathrm{km}$ (converted to $1064 \mathrm{~nm}$ ) [14]. The directional scattering ratio depends on the observed angle against polarisation direction and the angle between observed orientation and the incident direction as expected. The variation of the inhomogeneity is between $4.1 \%$ and $4.6 \%$. The bulk scattering level is thus not negligible compared to other optical losses such as absorption $\left(\sim 1 \mathrm{ppm} \mathrm{cm}^{-1}\right)$. Given the size of the substrates for present and planned gravitational wave detectors $(10-20 \mathrm{~cm})$ these losses are small compared to the losses due to the arm cavities mirror coatings ( $\sim 1 \mathrm{ppm}$ absorption and $\sim 10 \mathrm{ppm}$ scattering $[15,16])$ since the latter are amplified by the finesses of the Fabry-Perot cavities (several hundreds).

\section{Acknowledgment}

The authors thank Yanbei Chen for useful discussions. This project is supported by Australian Research Council and is part of the Australian Consortium for Interferometric Gravitational Astronomy.

\section{References}

[1] W. Winkler, K. Danzmann, A. Rüdiger, R. Schilling, Phys. Rev. A 44 (1991) 7022.

[2] S. Hild, H. Lück, W. Winkler, K. Strain, H. Grote, J. Smith, M. Malec, M. Hewitson, B. Willke, J. Hough, K. Danzmann, Appl. Opt. 45 (2006) 7269.

[3] F. Benabid, M. Notcutt, L. Ju, D.G. Blair, Opt. Commun. 167 (1999) 7.

[4] Z. Yan, C. Zhao, L. Ju, S. Gras, P. Barriga, D. Blair, Rev. Sci. Instrum. 76 (2005) 015104.

[5] Tauc Jan, in: S. Shashanka (Ed.), Optical Properties of Highly Transparent Solid, Mitra and Bernard Bendow, Plenum Press, 1975.

[6] D.A. Pinnow, T.C. Rich, F.W. Ostermayer, M. DiDomenico, Appl. Phys. Lett. 22 (1973) 527.

[7] L. Ju, X. Chen, Z. Yan, C. Zhao, D.G. Blair, J. Opt A: Pure App. Opt. 11 (2009) 125

[8] Z. Yan, L. Ju, S. Gras, C. Zhao, D.G. Blair, J. Opt A: Pure App. Opt. 6 (2004) 635.

[9] H. Grote and the LIGO Scientific Collaboration, Class. Quantum Grav. 27084003 (2010).

[10] T. Accadia et al., Class. Quantum Grav. 28114002 (2011).

[11] E.R. Pike, W.R. Pomeroy, J.M. Vaughan, J. Chem. Phys. 62 (8) (1975) 3188.

[12] K. Saito, H. Kakiuchida, A.J. Ikushima, J.Non-Cryst. Solids 222 (1997) 329.

[13] H. Utiyama, in: M.B. Huglin (Ed.), Light Scattering from Polymer Solutions, Academic, London, 1972, Chap. 4.

[14] J. Schroeder, R. Mohr, C.J. Montrose, P.B. Macedo, Journal of Non-Crystalline Solids $13(1973 / 74) 313$.

[15] L. Zhang, et al., http://www.ligo.caltech.edu/docs/G/G080162-00/G080162-00. pdf.

[16] F. Beauville, Virgo collaboration, Class. Quantum Grav. 21 (2004) S935. 